\title{
Isolation and Identification of Local Ethanol Tolerant Yeast Populating Distillation and Milling Sites in Nigeria
}

\author{
Gidado Rose Suniso Maxwell ${ }^{1,3,}$, , Etim Veronica Archibong ${ }^{2}$, Nweke Ogechi ${ }^{2}$, \\ Iloh Andrew Chibuzor ${ }^{2}$, Isu Rosemary Nennaya ${ }^{3}$, Solomon Bamidele Ogbe ${ }^{4}$ \\ ${ }^{1}$ Agricultural Biotechnology Department, National Biotechnology Development Agency (NABDA), Abuja, Nigeria \\ ${ }^{2}$ Biotechnology Advanced Research Center, Sheda Science and Technology Complex (SHESTCO), Abuja, Nigeria \\ ${ }^{3}$ Department of Biological Sciences, University of Abuja, Abuja, Nigeria \\ ${ }^{4}$ Department of Chemical Engineering, Obafemi Awolowo University, Ile-Ife, Nigeria
}

Email address:

roxydado91@gmail.com (G. R. S. Maxwell), roxydado@yahoo.com (G. R. S. Maxwell)

${ }^{*}$ Corresponding author

\section{To cite this article:}

Gidado Rose Suniso Maxwell, Etim Veronica Archibong, Nweke Ogechi, Iloh Andrew Chibuzor, Isu Rosemary Nennaya, Solomon Bamidele Ogbe. Isolation and Identification of Local Ethanol Tolerant Yeast Populating Distillation and Milling Sites in Nigeria. American Journal of BioScience. Vol. 4, No. 5, 2016, pp. 58-63. doi: 10.11648/j.ajbio.20160405.11

Received: April 24, 2016; Accepted: August 25, 2016; Published: September 12, 2016

\begin{abstract}
Two yeast strains referred to as OY and SY were isolated and characterised from local distillery and cereal milling sites. Isolation was done using potato dextrose media supplemented with $0.2 \%$ chloramphenicol. Morphological and biochemical results together with the rDNA internal transcribed spacer region (ITS) were identified as Pichia kudriavzevii strains GY1 and L9 respectively. OY and SY are ethanol tolerant strains, withstanding ethanol concentrations of up to $20 \%$ $(\mathrm{v} / \mathrm{v})$ in yeast extract, peptone, malt extract, glucose media. OY and SY displayed good growth in ethanol supplemented medium with $\mathrm{pH}$ ranging from $4.8-5.5$ at $30^{\circ} \mathrm{C}$. Growth measurements were determined by measuring optical density of the cells in broth using spectrophotometer at $570 \mathrm{~nm}$. The results obtained suggested that OY and SY demonstrated good parameters as ideal candidates for bioethanol production.
\end{abstract}

Keywords: Ethanol, Ethanol Tolerance, Saccharomyces, Non-saccharomyces, Pichia kudriavzevii

\section{Introduction}

The production of bioethanol through the conversion of reducing sugars such as glucose, sucrose and fructose by suitable microbial cells has seen explored for decades particularly due to the numerous industrial applications of ethanol [1]. Typically, species of Saccharomyces including Saccharomyces cerevisiae, Saccharomyces carlsbergensis, Saccharomyces diastaticus, etc are the most commonly used yeast cells as they are considered to be more robust and tolerant to ethanol than bacteria [2], [3], [4]. In addition, alcoholic fermentation processes leads to a decrease in oxygen solubility as the process temperature increases, thus microbial involvement requires candidates that function under anaerobic conditions. Furthermore, the preference for yeast over bacteria cells for ethanol production is due to the inherent ability of the former to withstand higher temperatures, grow at high osmotic pressure, yield higher levels of ethanol, etc [5].

Selection of new candidate yeast strains that can tolerate different levels of ethanol adds to the arsenal of microorganisms that can be manipulated for different industrially significant fermentation processes. Reports suggest that non-Saccharomyces yeasts like Candida $s p$., Hansenula sp., Kloeckera sp., Torulaspora sp., Kluyueromyces sp., Pachysolen tannophilus, Pichia stipitis and many more possess the ability to grow and participate in alcoholic fermentation [6], [7], [8].

Although the volume and concentration of ethanol increases as the fermentation process proceeds, thereby hindering the activity and growth of wild-type nonSaccharomyces yeast, it was conceptualized that only native 
or modified Saccharomyces would thrive under such conditions [9]. However, non-Saccharomyces yeast strains like Pichia anomala isolated from sugar rich environments was reported to endure ethanol concentrations of up to $14 \%$ [10]. Prior to that, studies indicated Saccharomyces sp., being the most ethanol tolerant yeast specie, possessed an average level of tolerance in the region of $12 \%(\mathrm{v} / \mathrm{v})$ ethanol, depending on the strain involved [11], [12]. As a mesophilic organism, Saccharomyces cerevisiae growth optimum is usually at $30^{\circ} \mathrm{C}, \mathrm{pH} 4.5-5.5$ [13].

Since our environment contains a large diversity of microorganisms, naturally evolved yeast strains may exist that possesses similar or higher levels of ethanol tolerance and growth optima at similar parameters. The objective of this study was to isolate, screen and characterize indigenous strains of yeast that possess the ability to tolerate selected concentrations of ethanol.

\section{Materials and Methods}

\subsection{Isolation of Yeast}

Sources selected for isolation of ethanol tolerant yeasts were soil samples from a local distillery in Odi local government area of Bayelsa state and also from wet cereal (millet, sorghum and corn) mill in Sheda village of Federal Capital Territory, Abuja, both in Nigeria. The source samples were serially diluted in sterile distilled water and inoculated on potato dextrose agar (PDA) medium plates by spread plate method. These plates were incubated at $30^{\circ} \mathrm{C}$ for 72 hours. After incubation developed colonies were observed for their morphology and microscopic characteristic. Colonies obtained from the isolation step were sub-cultured by streaking on PDA supplemented with $0.2 \mathrm{mg} / \mathrm{ml}$ chloramphenicol to inhibit bacterial growth. Colonies with morphologically distinct characters were observed and purified by repeat streaking on PDA medium. Pure culture of each strain was kept on potato dextrose agar slants and stored at $4{ }^{\circ} \mathrm{C}$ until needed for further studies. The obtained isolates were subjected to morphological identification with the following parameters: Colour, Shape, Texture, Elevation, Margin and Opacity and crosschecked using the methods of [14]. Wet mounts of isolated cell samples were prepared in distilled water and examined using 40X objective magnification.

\subsection{Identification by Fermentation Assessment}

The isolates obtained were analysed for their ability to utilize carbon sugars like fructose, glucose, galactose and sucrose, which also served as a method of biochemical identification using Phenol red broth (yeast fermentation base). $1 \mathrm{~g}$ of each sugar type was placed in a test tube containing $5 \mathrm{ml}$ of Phenol Red broth medium. After inoculating each tube with the test isolates, the tubes were incubated for 48 hours at $30^{\circ} \mathrm{C}$. A colour change from red to yellow due to acid production gave a positive indication of their fermentative capability [14].

\subsection{Screening for Ethanol Tolerance}

The yeast isolates were inoculated unto $10 \mathrm{mls}$ of YPMG broth (yeast extract, peptone, malt extract, glucose) supplemented with different concentrations of ethanol $(0,5$, $10,15,20 \% \mathrm{v} / \mathrm{v})$ differing by $5 \%(\mathrm{v} / \mathrm{v})$ from one flask to another. The tubes were incubated for 120 hours at $30^{\circ} \mathrm{C}$. Samples were drawn every 24 hours and growth was measured using a UV-VIS spectrophotometer at $570 \mathrm{~nm}$. Also, the viability of yeast cells were checked inoculating the isolates unto $10 \mathrm{ml}$ of YPMG broth supplemented with different concentrations $(0,5,10,15$ and $20 \% \mathrm{v} / \mathrm{v})$ of ethanol. Viability was measured following 48 hours of incubation at $30^{\circ} \mathrm{C}$ by serially diluting with sterile distilled water and plated on YPMG agar medium. The number of $\mathrm{CFU} / \mathrm{ml}$ was calculated [14]. Variations in $\mathrm{pH}$ was also monitored in broth cultures.

\subsection{Molecular Characterization}

Isolates were genetically identified via amplification and sequence analysis of the ribosomal DNA internal transcribed spacer region (ITS) [15]. The primers; ITS1 (5 1 TCCGTAGGTGAACCTGCGG- $\left.3^{1}\right)$ and ITS4 (5 4 TCCTCCGCTTATTGATATGC- $3^{1}$ ), were used for $\mathrm{rDNA}$ amplification. The amplification reaction was performed in a final volume of 50 micro litre containing $10 \mathrm{mM}$ Tris- $\mathrm{HCl}$, $50 \mathrm{mM} \mathrm{KCl}, 1.5 \mathrm{mM} \mathrm{MgCl}_{2}$, each dNTPs at a concentration of $0.2 \mathrm{mM}, 1.25 \mathrm{IU}$ of Taq polymerase, each primer at a concentration of $0.2 \mathrm{mM}$ and 10 micro litre of DNA (50ng) template.

The reactions were run for 36 cycles with denaturation at $94^{\circ} \mathrm{C}$ for $2 \mathrm{~min}$, annealing at $52^{\circ} \mathrm{C}$ for $1 \mathrm{~min}$ and elongation at $72^{\circ} \mathrm{C}$ for $2 \mathrm{~min}$. The PCR products were purified using the Nucleic Acid and Protein purification Kit and cloned into the pGEM vector then sequenced. Sequences of the PCR product were compared with known ITS region sequences deposited in the GenBank (http://www.ncbi.nlm.nih.gov) and the percentage of similarity among the fragments was calculated using the BLAST program (http://www.ncbi.nlm.nih.gov/BLAST/).

\section{Results}

\subsection{Isolation and Identification of Isolates}

Two yeast strains obtained from the successful isolation from the studied sites (Odi and Sheda) were given the designation OY and SY respectively. Morphological examination of these two isolates revealed a creamy white, round shaped, smooth textured and flat elevation with transparent opacity (figure 1, table 1). The budding stage of the yeast isolates was observed under (40X) microscope (Table 1).

In the assessment of its fermentation ability for a select number of sugar sources, the two isolates were proficient in utilizing all tested sugars; fructose, glucose, galactose and sucrose using phenol red broth assay (Table 2). The 
morphological and sugar fermentation data led to the preliminary suspicion that the isolates were strains of Saccharomyces cerevisiae.

Figure 2 was obtained from the analysis of amplified DNA fragments, approximately $600 \mathrm{bp}$ in size, from the yeast isolates. The partial nucleotide sequences obtained were subjected to BLAST analysis and the identity was established on the basis of sequence similarity and closest neighbour (figure 3). The blast sequence query showed that Pichia kudriavzevii strains GY1 and L9 (JQ808004.1 and KF806465.1) respectively from the Genbank Library database, has $97 \%$ and $98 \%$ sequence homology with the genomic DNA sequence of OY and SY, respectively at both ITS regions (Table 3 ).

\subsection{Ethanol Tolerance of Yeast Isolates}

The effect of ethanol on the growth rate of OY and SY is depicted in figure 4. Both isolates grew well in the presence of $5 \%$ ethanol. From $10-20 \% \quad(\mathrm{v} / \mathrm{v})$ exogenous ethanol presence, growth still continued although inhibition was present was inhibited. Table 4 indicates the $\mathrm{CFU} / \mathrm{ml}$ obtained from broth cultures.

\section{3. pH Analysis}

$\mathrm{pH}$ measurements within the broth over the period of incubation with increasing concentrations of ethanol was examined (table 4). Based on the data obtained, fluctuations in $\mathrm{pH}$ levels were observed roughly between $\mathrm{pH}$ 5.1-5.5 in samples broth without any initial ethanol. The expected differences over the time course may address the integrity of the cell membrane in addition to the organisms' acidification activity.
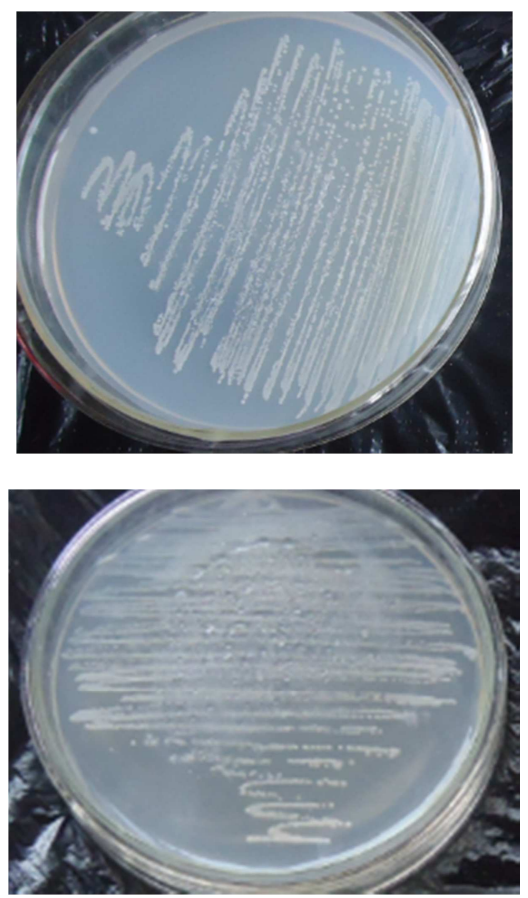

Figure 1. Pictures of pure yeast isolates on PDA medium. Top = strains obtained from Odi, Bottom = strains obtained from Sheda.

Table 1. Morphological identification of yeast isolates.

\begin{tabular}{|c|c|c|c|c|c|c|c|}
\hline Yeast Strain & Colour & Shape & Texture & Elevation & Margin & Opacity & Bud Presence \\
\hline OY & Creamy white & Round & Smooth & Flat & Entire & Transparent & + \\
\hline SY & Creamy white & Round & Smooth & Flat & Entire & Transparent & + \\
\hline
\end{tabular}

Table 2. Sugar fermentation using Phenol Red broth test.

\begin{tabular}{llllll}
\hline Yeast Strain & Fructose & Galactose & Glucose & Sucrose & Suspected organisms \\
\hline OY & + & + & + & + & Saccharomyces cerevisiae \\
SY & + & + & + & + & Saccharomyces cerevisiae \\
\hline
\end{tabular}

Key: +=positive, -=negative

Table 3. Identification of yeast isolates based on sequence alignment (BLAST).

\begin{tabular}{lllll}
\hline Culture code & Nearest phylogenetic relative & Strain & Accession number & Sequence similarity (\%) \\
\hline OY & Pichia kudriavzevii & GY1 & JQ808004.1 & 97 \\
SY & Pichia kudriavzevii & L9 & KF806465.1 & 98 \\
\hline
\end{tabular}

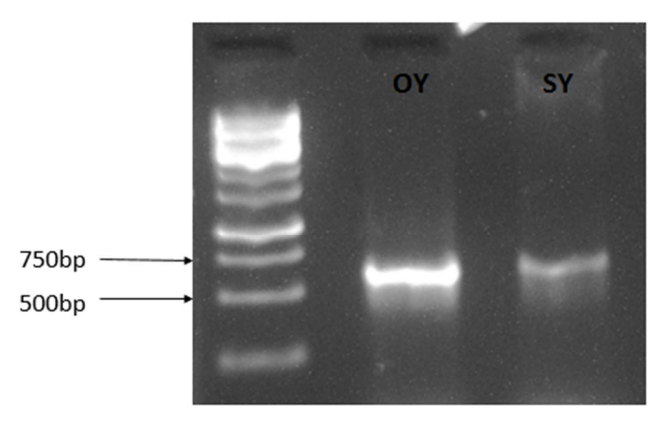

Figure 2. Gel electrophoresis micrograph of amplified products.

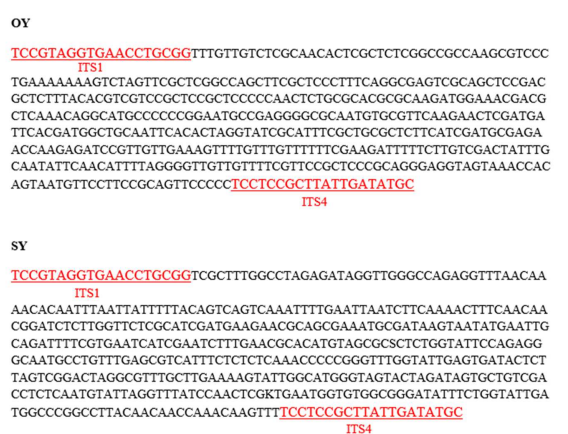

Figure 3. Sequencing results of the ITS region of Y-1. Primer sequences were underlined. 


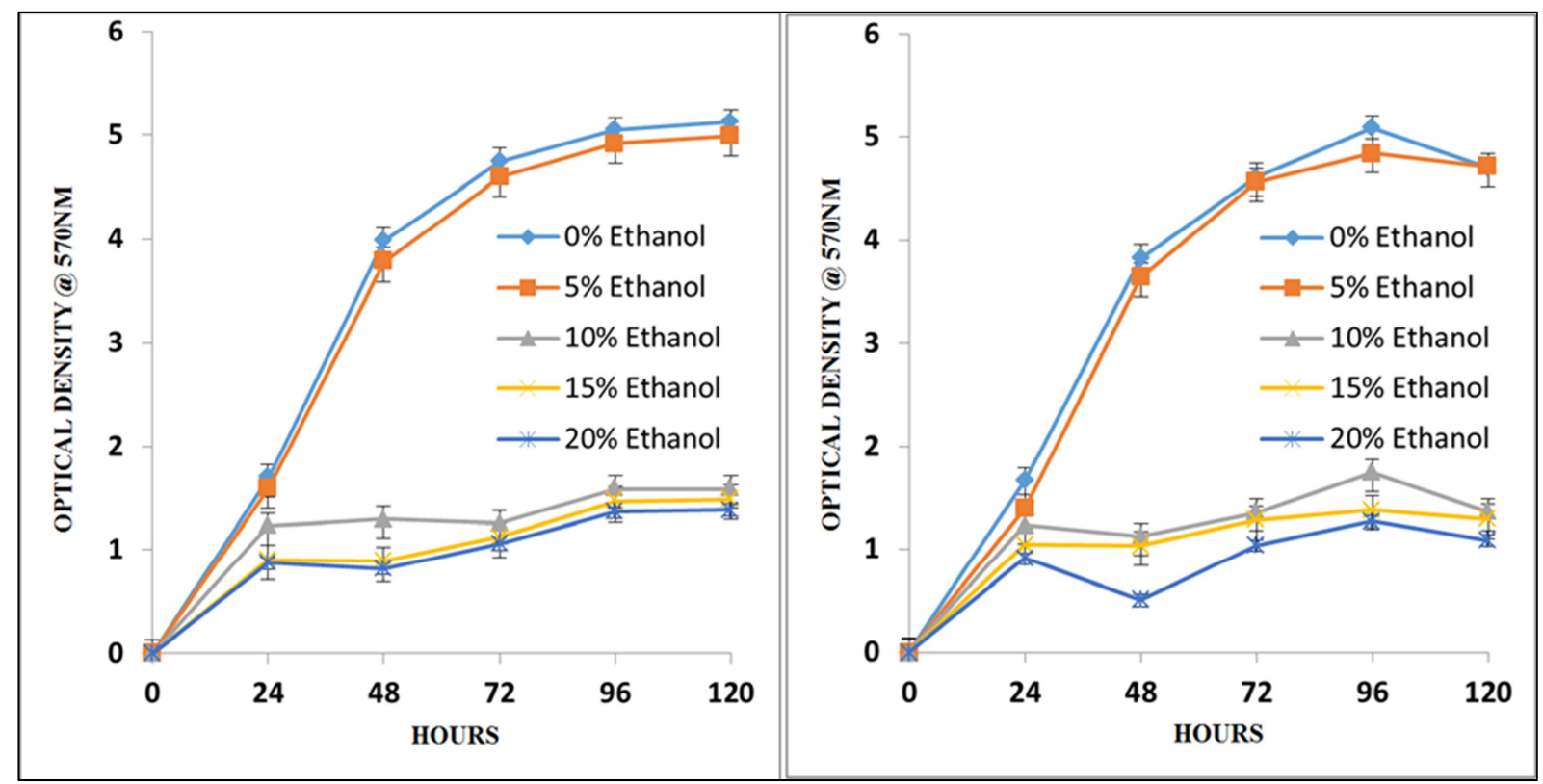

Figure 4. Comparative growth determination of OY (left) and SY (right) over time with increasing concentrations (\%, v/v) of ethanol. Data was obtained from the mean triplicates.

Table 4. pH read-out for isolates $O Y$ and $S Y$.

\begin{tabular}{lllllllll}
\hline OY & & & & SY & & \\
\hline \% Ethanol (v/v) & $\mathbf{2 4}(\mathbf{h r})$ & $\mathbf{4 8}(\mathbf{h r})$ & $\mathbf{7 2} \mathbf{( h r})$ & $\mathbf{9 6}(\mathbf{h r})$ & $\mathbf{2 4}(\mathbf{h r})$ & $\mathbf{4 8}(\mathbf{h r})$ & $\mathbf{7 2}(\mathbf{h r})$ & $\mathbf{9 6}(\mathbf{h r})$ \\
\hline 0 & $5.3 \pm$ & $5.2 \pm$ & $5.4 \pm$ & $5.3 \pm$ & $5.2 \pm$ & $5.1 \pm$ & $5.4 \pm$ & $5.5 \pm$ \\
5 & $5.1 \pm$ & $5.0 \pm$ & $5.3 \pm$ & $5.3 \pm$ & $5.0 \pm$ & $5.0 \pm$ & $5.3 \pm$ & $5.4 \pm$ \\
10 & $5.0+$ & $5.0 \pm$ & $5.2 \pm$ & $5.3 \pm$ & $4.9 \pm$ & $4.9 \pm$ & $5.3 \pm$ & $5.2 \pm$ \\
15 & $4.0 \pm$ & $5.0 \pm$ & $5.2 \pm$ & $5.2 \pm$ & $4.9 \pm$ & $4.8 \pm$ & $5.3 \pm$ & $5.2 \pm$ \\
20 & $4.9 \pm$ & $5.0 \pm$ & $5.2 \pm$ & $5.1 \pm$ & $4.9 \pm$ & $4.8 \pm$ & $5.2 \pm$ & $5.2 \pm$ \\
\hline
\end{tabular}

\section{Discussion}

For over a decade, suggestions that naturally occurring non-Saccharomyces yeasts like Candida, Hansenula, Kloeckera, and Torulaspora possess the ability to thrive under different environmental conditions and participate in a number of industrial processes has fuelled rediscovery of other microbial species from uncommon environments with the hope of tapping into the vast resources from such environments whilst boosting our collective knowledge of microbial life on earth [6], [17]. Selected advances in biotechnology like metabolic pathway engineering have boosted both the yield and improvement of product formation using microorganisms. While approaches like this would require computer modelling, targeted mutagenesis and gene disruption techniques, the isolation of naturally evolved microbial isolates from which enhanced quality and quantity of industrially relevant compounds as well as lower production process costs is an economically viable attempt to deliver useful products to mankind [18].

Proper identification of some fungal species still possesses a challenge owing to structural changes which results in the generation of false positives when using only biochemical and phenotypic techniques of identification
(However, the advent of DNA-based methods largely overcame the limitations of traditional methods and studies using molecular approaches revealed a greater diversity in fungi [19].

It has been reported that based on biochemical and morphological characterization alone, strains of Candida albicans, Candida famata, Candida palmioleophila, Debaryomyces hansenii and Pichia guilliermondi have all been misidentified at one point in research due to a high degree of similarity [20]. Even with the availability of commercial identification kits (API®20C AUX, Biomérieux, France) reports indicate that the issue of inconsistent identification still persists. The DNA-based method for taxanomic identification serves as the most ideal method for yeast identification from a pool of morphologically similar yet genetically diverse cultures using the conserved ITS-5.8S regions of the ribosomal cistron.

In this investigation, the distinct colonies obtained from the antibiotic supplemented potato dextrose agar culture were observed to be creamy white in colour, smooth surfaced with a flat elevation as well as possessing a transparent opacity with no pseudo-hypha formation (Fig. 1, Table 1).

Preliminary biochemical investigation revealed that all four cultured strains possessed the ability to utilize carbon sugars like fructose, glucose, galactose and sucrose using 
Phenol red broth (yeast fermentation base). The colour change observed (red to yellow) was indicative of the presence of an array of organic acids which may include phytic acid, a compound recently reported to be produced by Pichia species and a number of other non-Saccharomyces yeasts including Arxula adeninivorans, Candida krusei, Cryptococcus laurentii, Debaryomyces castellii, Debaryomyces occidentalis, Pichia sp., Rhodotorula gracilis, Schwanniomyces castellii, etc which aids in the release of phosphorus compounds from organic sources in the soil [21]. This observation suggests that the culture organisms possess good fermentative capabilities. This result is similar to that of [22] who observed that yeast isolates studied could ferment one or more sugar types. The respective sequences for each isolate, obtained via PCR amplification using the ITS1 and ITS4 primer combinations, cloning and sequencing were queried against the (nr) nucleotide bank using the BLAST program (Figure 3). The nucleotide BLAST query revealed that all isolates share extremely high/complete identity with corresponding sequences that are deposited in GenBank; samples OY and SY sequences corresponded to that of Pichia kudriavzevii strains GY1 and L9 (97\% and 98\% respectively). These two isolates obtained share close homology with Issatchenkia orientalis, Saccharomyces krusei and Candida guilliermondii, the later which is amorphous to Pichia sp. The data obtained from this study supports the use of DNA-based identification to avoid misidentification. Yeast participation in fermentation is limited by the inhibitory effects of ethanol concentration which increases over the course of fermentation. The adoption of new or emerging yeast isolates towards large scale ethanol production relies upon its ability to tolerate certain levels of ethanol within the broth [23]. Since studies indicated that the generally accepted benchmark for ethanol tolerance amongst yeast isolates is $12 \%(\mathrm{v} / \mathrm{v})$ ethanol, the two isolates in this study; OY and SY (Pichia kudriavzevii) displayed continuous cell growth up to $20 \%$ (v/v) (figure 4).

At an optimum temperature of $30^{\circ} \mathrm{C}$, variation in $\mathrm{pH}$ was accessed in broth cultures incubated over 96 hours, with different concentrations of ethanol. The results were shown in table 4. Based on the data obtained, fluctuations in $\mathrm{pH}$ levels were observed roughly between $\mathrm{pH}$ 5.1-5.5 in sample broths without any initial ethanol. The expected differences over the time course may address the integrity of the cell membrane in addition to the organisms' acidification activity. Since the $\mathrm{pH}$ optima for both inhibited and uninhibited ethanol fermentation is reported to be about 5.5, the data in this study is in line with established data [24]. In the broth cultures containing varying concentrations of ethanol, there seems to be an addition metabolic by-product that may account for differences in energetic level that helped the cells maintain their physiological state. The formation of weakly acidic acids like acetic and formic acids, both of which are lipo-soluble could diffuse across plasma membranes of yeast cells and raises the intracellular $\mathrm{pH}$. To thrive under such conditions, it is conceivable that the yeast cells would retort to the diffusion process by expending ATP to repair the membrane and maintain a constant intracellular $\mathrm{pH}$ [25], [26]. From the data obtained (table 4), Pichia kudriavzevii appears to be an acidophilic organism and grows better under acidic conditions with an optimal $\mathrm{pH}$ range varied from $\mathrm{pH}$ 4-6. The intracellular enzymes of this yeast seems to work best at its optimal $\mathrm{pH}$ it leads to maximal conversion of sugar into ethanol.

The present study has revealed the availability of indigenous non-saccharomyces yeast species that possess tolerance to ethanol therefore presenting alternative candidates for ethanol production, thus reaffirming data obtained in another study [27].

\section{Conclusion}

The data collected from this study show that non Saccharomyces yeast; Pichia kudriavzevii solated from sugar rich environments were able to tolerate ethanol concentrations of up to $20 \%(\mathrm{v} / \mathrm{v})$. These indigenous isolates function efficiently at $\mathrm{pH} 5-6$, and temperature $30^{\circ} \mathrm{C}$. The results obtained from this study support the use of other native non Saccharomyces yeast species in the production of ethanol, utilizing locally available materials.

\section{Acknowledgements}

The authors are grateful to the DG/CEO Sheda Science and Technology Complex (SHESTCO), and the Director of the Biotechnology Advanced Research Centre, (SHESTCO) Abuja, Nigeria for their support. The authors would like to thank Mr. Godwin Etuk-Udo and Mrs. Oluwa Kemi Olatilu of the Biotechnology Advanced Research Centre, Sheda Science and Technology Complex (SHESTCO) for useful technical discussions.

\section{References}

[1] Farrell, A. E., Plevin, R. J., Turner, B. T., Jones, A. D., O'Hare, M., and Kammen, D. M. (2006). Ethanol can contribute to energy and environmental goals. Science, 311: 506- 508.

[2] Davis, L., Rogers, P., Pearce, J., and Peiris, P. (2006). Evaluation of Zymomonas based ethanol production from a hydrolysed waste starch stream. Biomass and Bioenergy, 30: 809-814.

[3] Edgardo, A., Parra, C., Manuel, R., Juanita, F., Baeza, J. (2008). Selection of thermotolerant yeast strains Saccharomyces cerevisiae for bioethanol production. Enzyme and Microbial Technology, 43: 120-123.

[4] Signori, L., Passolunghi, S., Ruohonen, L., Porro, D., and Branduardi P. (2014). Effect of oxygenation and temperature on glucose-xylose fermentation in Kluyveromyces marxianus CBS712 strain. Microbial Cell Factories, 13: 51-64.

[5] Cakar Z., Seker U., Tamerler C., Sonderegger, M., and Sauer, U. (2005). Evolutionary engineering of multiple-stress resistant Saccharomyces cerevisiae. FEMS Yeast Research, 5: 569-578. 
[6] Jolly, N. P., Augustyn, O. P. H., and Pretorius, I. S. (2003). The effect of non-Saccharomyces yeasts on fermentation and wine quality. South African Journal of Enology and Viticulture, 24: 55-62.

[7] Matsushika A., Inoue, H., Kodaki, T., and Sawayama, S. (2009). Ethanol production from xylose in engineered Saccharomyces cerevisiae strains: current state and perspectives. Applied Microbiology and Biotechnology, 84: $37-53$.

[8] Ciani, M., Comitini, F., Mannazzu, I., and Domizio, P. (2010). Controlled mixed culture fermentation: a new perspective on the use of non-Saccharomyces yeasts in winemaking. FEMS Yeast Research, 10: 123-133.

[9] Li, J. R., and Cai, A. Q. (2007). Isolation and identification of main microorganisms in traditional distiller's yeast. LiquorMaking Science and Technology, 5: 111-115.

[10] Tao N., Gao, Y., and Liu, Y., (2011). Isolation and characterization of a Pichia anomala strain: a promising candidate for bioethanol production. Brazilian Journal of Microbiology, 42: 668-675.

[11] Cocolin, L., Bisson, L. F., and Mills, D. A. (2000). Direct profiling of the yeast dynamics in wine fermentations. FEMS Microbiology Letters, 189: 81-87.

[12] Tofalo, R., Chaves-Lopez, C., Fabio, F. D., Schirone, M., Felis, G. E., Torriani, S., et al. (2009). Molecular identification and osmotolerant profile of wine yeasts that ferment a high sugar grape must. International Journal of Food Microbiology, 130: 179-187.

[13] Bhima, B., Marrivada, S. R., Devi, T. A., Reddy, Y. R., and Rao, L. V. (2010). Screening and characterization of stress tolerant Saccharomyces Cerevisiae isolated from brewery effluents for animal probiotic applications. IIOAB Research: Food Bio-technology, 1: 32-38.

[14] Barnett, J. A., Payne, R. W., and Yarrow, D. (2000). Yeasts: Characteristics and identification. 3rd edn. Cambrie University Press, UK. ISBN-13: 978-0521573962.

[15] Khaing, T. W., Weine, N., and Mya, MO. (2008). Isolation, Characterization and Screening of Thermo tolerant, Ethanol Tolerant Indigenous Yeasts and Study on the Effectiveness of Immobilized Cell for Ethanol Production. Journal of Science and Technology, 1: 12-14.

[16] Caggia, C., Restuccia, C., Pulvirenti, A., and Giudici, P. (2001). Identification of Pichia anomala isolated from yoghurt by RFLP of the ITS region. International Journal of Food Microbiology, 71: 71-73.

[17] Lee, Y. J., Choi, Y. R., Lee, S. Y., Park, J. T., Shim, J. H., Park, K. H., and Kim, J. W. (2011). Screening Wild Yeast Strains for Alcohol Fermentation from Various Fruits. Mycobiology 39: 33-39.
[18] Barrio, E., González, S. S., Arias, A., Belloch, C., and Querol, A. (2006). Molecular mechanisms involved in the adaptive evolution of industrial yeasts. In: Yeasts in Food and Beverages (Eds: Querol A, Fleet G). Springer-Verlag, Berlin, Germany. pp 153-173.

[19] Chen, Y. C., Eisner, J. D., Kattar, M. M., Rassoulian-Barrett, S. L., LaFe, K., Yarfitz, S. L., Limaye, A. P., and Cookson, B. T. (2000). Identification of medically important yeast using PCR-based detection of DNA sequence polymorphism in the internal transcribed spacer 2 region of the rRNA genes. Journal of Clinical Microbiology, 38: 2302-2310.

[20] Desnos-Ollivier, M., Ragon, M., Robert, V., Raoux, D., Gantier, J. C., and Domer, F. (2008). Debaryomyces hansenii (Candida famata), a rare human fungal pathogen often misidentified as Pichia guilliermondii (Candida guilliermondii). Journal of Clinical Microbiology, 46: 32373242 .

[21] Mukherjee, V., Steensels, J., Lievens, B., et al. (2014). Phenotypic evaluation of natural and industrial Saccharomyces yeasts for different traits desirable in industrial bioethanol production. Applied Microbiology and Biotechnology, 98: 9483-9498.

[22] Walker, L. P., Hii, H., and Wilson, D. B. (2006). Enzymatic hydrolysis of cellulose: An Overview. Bioresources Technology, 36: 3-14.

[23] Lei, J. J., Zhao, X. Q., Ge, X. M., and Bai, F. W. (2007). Ethanol tolerance and the variation of plasma membrane composition of yeast floc populations with different size distribution. Journal of Biotechnology, 131: 270-275.

[24] Rajoka, M., Khalid A, and Ferhan, M. (2005). Kinetic and thermodynamics of ethanol production by a thermotolerant mutant of Saccharomyces cerevisiae. Letters in Applied Microbiology, 40: 316-21.

[25] Wang, Z. X., Zhuge, J., Fang, H., and Prior, B. A. (2001). Glycerol production by microbial fermentation: A review. Biotechnology Advances, 19: 201-223.

[26] Thomas, K. C., Hynes, S. H. and Ingledew, W. M. (2002). Influence of medium buffering capacity on inhibition of Saccharomyces cerevisiae growth by acetic and lactic acids. Applied and Environmental Microbiology, 68: 1616-1623.

[27] Ruyters, S., Mukherjee, V., Verstrepen, KJ., et al. (2015). Assessing the potential of wild yeasts for bioethanol production. Journal of Industrial Microbiology and Biotechnology, 42: 39-48. 